\title{
Electric Space Heating Scheduling for Real-time Explicit Power Control in Active Distribution Networks
}

\author{
Giuseppe Tommaso Costanzo*, Andrey Bernstein ${ }^{\dagger}$, Lorenzo Reyes Chamorro ${ }^{\ddagger}$, \\ Henrik W. Bindner*, Jean-Yves Leboudec ${ }^{\dagger}$ and Mario Paolone ${ }^{\ddagger}$ \\ ${ }^{*}$ Center for Electric Energy and Power, Technical University of Denmark, Campus Ris $\emptyset$ \\ Frederiksborgvej 399, 4000 Roskilde, Denmark. email:\{guco, hwbi\}@elektro.dtu.dk. \\ ${ }^{\dagger}$ Laboratory for Communications and Applications (LCA), Ecole Polytechnique Federale de Lausanne, \\ Route Cantonale, 1015 Lausanne, Switzerland. email:\{andrey.bernstein, jean-yves.leboudec\}@epfl.ch. \\ ${ }^{\ddagger}$ Distributed Energy Systems Laboratory (DESL), Ecole Polytechnique Federale de Lausanne, \\ Route Cantonale, 1015 Lausanne, Switzerland. email:\{lorenzo.reyes, mario.paolone\}@epfl.ch.
}

\begin{abstract}
This paper presents a systematic approach for abstracting the flexibility of a building space heating system and using it within a composable framework for real-time explicit power control of microgrids and, more in general, active distribution networks. In particular, the proposed approach is developed within the context of a previously defined microgrid control framework, called COMMELEC, conceived for the explicit and real-time control of these specific networks. The designed control algorithm is totally independent from the need of a building model and allows exploiting the intrinsic thermal inertia for real-time control. The paper first discusses the general approach, then it proves its validity via dedicated simulations performed on specific case study composed by the CIGRE LV microgrid benchmark proposed by the Cigré TF C6.04.02.
\end{abstract}

Index Terms-Demand-side Management, Explitic power control, Microgrids.

\section{INTRODUCTION}

Today's trend of vast penetration of distributed generation in low and medium voltage power networks threats the energy/power equilibrium as well as the quality-of-supply of these electrical grids in a way that, in several countries, operational constraints are already attained. Additionally, their active contribution to the operation of transmission networks needs to be re-defined. In particular, the main concerns of power transmission networks operators refer to the definition of new control schemes able to evaluate, and schedule, the contributions of these active networks to the grid ancillary services (with particular reference to voltage and frequency controls, e.g. [1]). It this respect, a fully-composable framework for explicit and real-time control of network power flows has been introduced in [2], where real-time means with a typical response time of $0.1 \mathrm{sec}$. It is based on three main elements: (i) an abstract framework that applies to electrical subsystems and specifies their capabilities, expected behaviour and a simplified view of their internal state; (ii) the agents responsible for subsystems/resources capable to communicate with other agents by using a simple, yet powerful, protocol and (iii) the recursive property, namely it is possible to compose a set of interconnected elements into a simple entity that responds to protocol messages (see [2] for further details). This framework allows for abstracting devices status and consumers or producers operation capabilities regardless of their type and operation mode. Device abstraction and control is performed by the local resource controller, which exposes a standard interface toward the grid controller. The grid controller is totally generic and needs information only on the grid topology and its status, as well as of the devices underneath, to compute the power setpoints for all the resources, thus guarantee the network safe operation. Such framework, called COMMELEC [2], is based on request/response communication protocol between the devices controllers (Resource Agents, RAs) and the grid controller (Grid Agent, GA). The RAs export generic and standardized information about devices status to the GA that computes, in real-time, the optimal power injections at the grid nodes that the various RAs are requested to realize. Thus, the design of a Resource Agent is specific to device type, whereas the GA is totally generic and can be adapted to any network.

Within the context of the COMMELEC framework, there is the interest in investigating whether the flexibility related to this type of load can be exploited. [2] has already presented the design of RAs for induction motors, PV plants and micro hydro turbines, while the building consumption has been treated as a non-controllable load. This paper goes further: it proposes a systematic approach for abstracting and representing the intrinsic flexibility related to building thermal inertia within the COMMELEC framework. The design of a RA for electric space heating system, called Load Agent (LA), is here presented. It uses the measured air temperature in different building sectors to compute the system flexibility and the other information sent to the GA.

The structure of the paper is the following: Section II summarizes the information exchange protocol and the COMMELEC framework by making use of a case study composed by the Cigré LV microgrid benchmark of the TF C6.04.02 [3]. In this first case study, the building space heating is a noncontrollable load. The simulation reported in this section shows the positive impact on the network performances that COMMELEC has with respect to the standard droop-based 
control approaches. From this starting point, the design of the Load Agent for electric space heating is fully explored in Section III. A simulation study using the building as flexible load is presented in Sec. IV. The simulation results quantify the positive impact of the proposed LA in improving the microgrid performances while increasing the production from renewables, all without any modification of the GA that does not need to be aware of the load under control of the LA. Sec. V outlines the final conclusions and the next research steps.

\section{THE COMMELEC FRAMEWORK}

In this section, we briefly discuss the COMMELEC framework for real-time control of electrical grids using explicit power setpoints introduced in [2]. This framework uses a hierarchical representation of software agents, each associated with a single device or an entire subsystem (including a grid and/or a number of devices). Thanks to a standardized interface between the agents, it is possible to completely disregard devices details in the overall network control and easily upscale (or downscale) the entire architecture. Each agent speaks for and controls the subsystem under its responsibility, which can consist of other electrical grids or resources (loads, generators and storage devices), by using explicit power setpoints.

An agent can be assigned the role of leader of one or more other agents, which we term the followers of that leader. The roles follow the topological/voltage hierarchy of power networks. An example of agents hierarchy is shown in Fig. 1, where there are two Grid Agents: one for the MV grid (called MVGA), and one for the LV grid (called LVGA). The LVGA is the leader of a group of Resource Agents (RAs).

The considered network model is mainly taken from [3], where a $0.4 k \mathrm{~V}$ benchmark microgrid is proposed. In [2] we have assumed to connect the low voltage microgrid with a simplified $20 \mathrm{kV}$ medium voltage grid. The whole network is considered islanded. As typically used in a microgrid setup, we assume that power electronic devices are used to interface the generation/storage units with the grid and, as presented in [2], the distributed generation is composed by (i) photovoltaic plants (PVi) and a hydraulic microturbine $(\mu \mathrm{H})$, (ii) two battery-based energy storage systems (ESS and ESS1), (iii) uncontrollable loads (ULi) and (iv) controllable loads modelled as water boilers (WBi) capable of deploying explicit power control setpoints (in the following, called just setpoints).

The agents communicate with each other by using a simple Advertisement/Request protocol, and using some simplified quantitative information about their devices capabilities and internal states. In particular, the follower agents periodically advertise to their leader the following three elements: (i) $P Q t$ profile, that is a region in $P Q t$ space of setpoints that the subsystem is willing to implement; (ii) Virtual cost function, that acts as proxy for the internal state of the system, the preferred setpoints, and how close the subsystem is to its operational constraints; and (iii) Belief function, representing all the possible values of $(P, Q)$ that the subsystem may

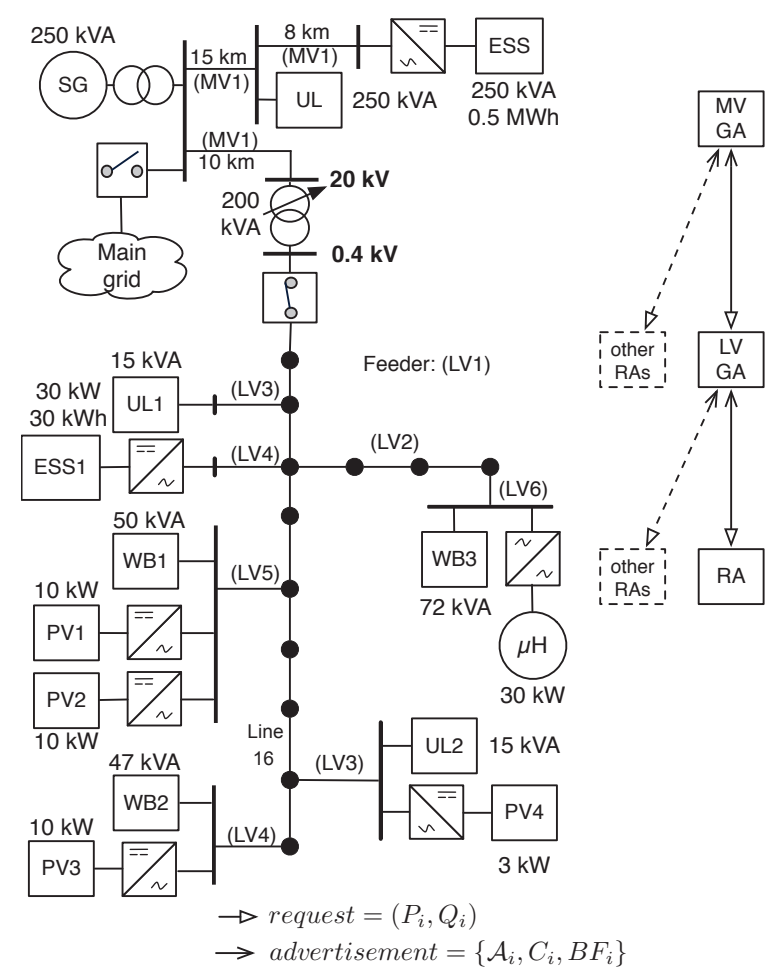

Fig. 1: Reference distribution network. The LV part of the grid is composed by the CIGRE LV microgrid benchmark proposed by TF C6.04.02 [3]. The MVGA is the leader of one or more LVGA, which themselves are leaders of one or more RAs (dotted arrows).

implement in practice when it receives a certain setpoint. Note that the latter is a set-valued function from $\mathbb{R}^{2}$ to sets over $\mathbb{R}^{2}$. Observe the difference between $P Q t$ profiles and belief functions: the former indicates the setpoints that this subsystem is willing to receive, whereas the latter indicate all the possible operating conditions that may result from applying a received setpoint. The $P Q t$ profile is used by the grid agent to compute an "optimal" setpoint, whereas the belief function is used to determine the region of the safe operation of the grid.

All agents monitor in real time the state of the grid device(s) and/or the agents (the GAs) under their responsibility and compute the general messages to send to their leader agent. When receiving a new request, each agent projects the power setpoint to the current updated $P Q t$ boundaries in order to ensure its individual safety. We refer the reader to [2] for further details on the protocol and the computation of the setpoints of the GA.

In the following we show the operation of COMMELEC and its positive impact in a $0.4 k \mathrm{~V} \mathrm{LV}$ microgrid in terms of: (i) minimization of renewables curtailment, (ii) local power balance, (iii) automatic management of the line congestions and voltage control in a case study where three different control methods are compared: COMMELEC, droop with only primary frequency control (DP) for all resources and primary voltage control only in the slack resource (in our case the ESS), and Droop with additional secondary frequency control 
(DPS) at the slack resource. The focus here is on the dynamic short-term behaviour, so that results are presented over a time horizon of 1600 seconds.

As it can be seen from Fig. 2, COMMELEC allows higher energy production from PV with respect to both droop control strategies. Figure $3 \mathrm{~b}$ shows COMMELEC performance in

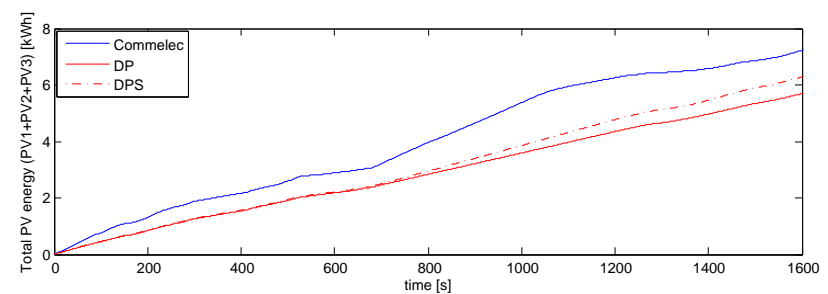

(a)

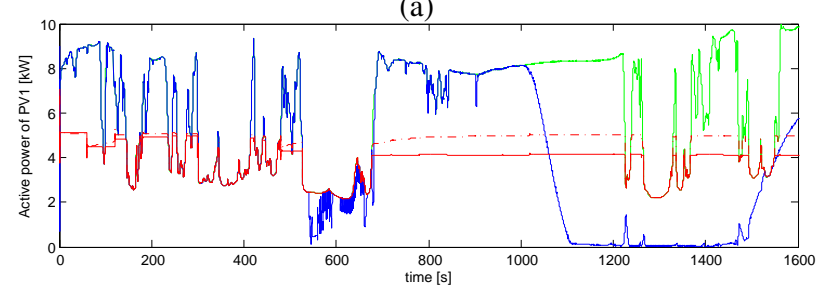

(b)

Fig. 2: Comparison between COMMELEC and Doop control strategies: total PV production (a) and detailed production of PV1 (b) (the green line is the ideal production).

controlling the local water boiler WB2 for compensating the local PV3 production, while the line current and node voltage are kept within the safe bounds (Fig. 3d). This case study shows some of the benefits introduced by COMMELEC, as the maximization of production from renewables and local power balance at LV level. Observe that in the network topology shown in Fig. 1 there is an uncontrollable load of $15 \mathrm{kVA}$ (UL2) connected at the end of the feeder. In the following study we replace this load with a $8 \mathrm{~kW}$ residential load consisting of building space heating. We next focus on the design of the Load Agent for this resource, while the grid configuration, the GA and the other agents remain unchanged.

\section{THE LOAD AGENT}

The algorithm, which design is here presented, is the core of the building Resource Agent, henceforth called Load Agent (LA), which generality and model-independent design this section is intended to enlighten. The presented LA can be adapted to buildings of any size and type provided that the heating system is electric (resistive heaters) and air temperature measurements are available in each control volume. Note that, since this study is based on simulations, the LA communicates with a building emulator (Fig. 4), which model is detailed in [4].

As introduced in Sec. II, the information the LVGA sends to RAs is an explicit power setpoint. RAs, as acknowledgement, send to the GA the $P Q t$ profile, the belief function and the virtual cost function, which calculation is presented in the following three subsections. The fourth subsection presents

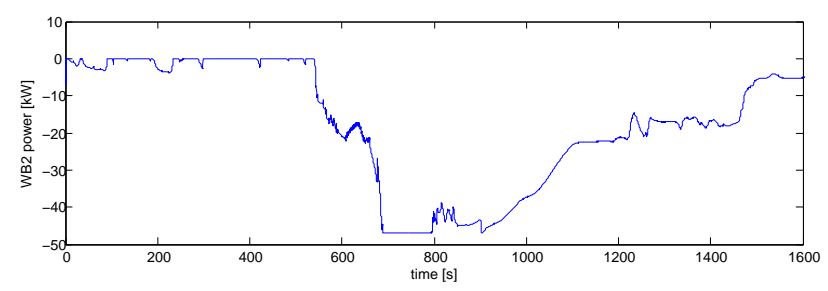

(a)

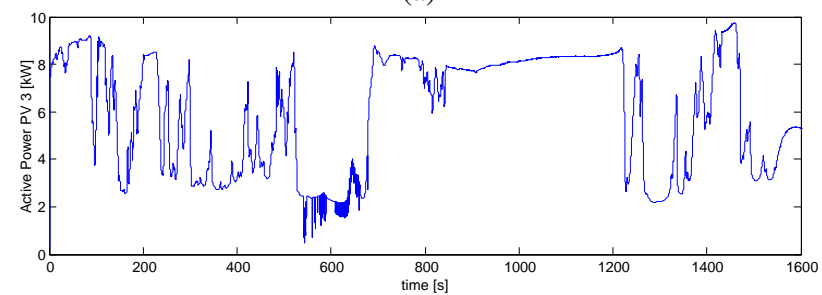

(b)

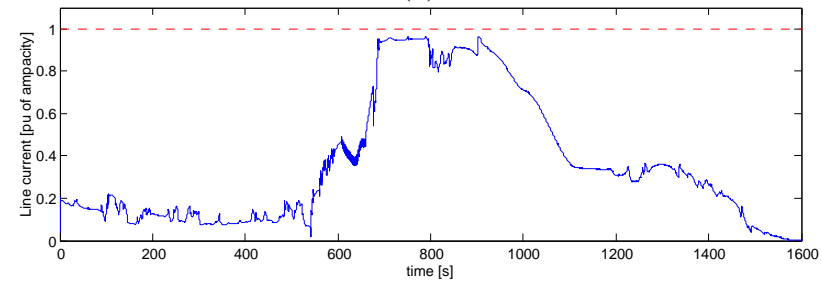

(c)

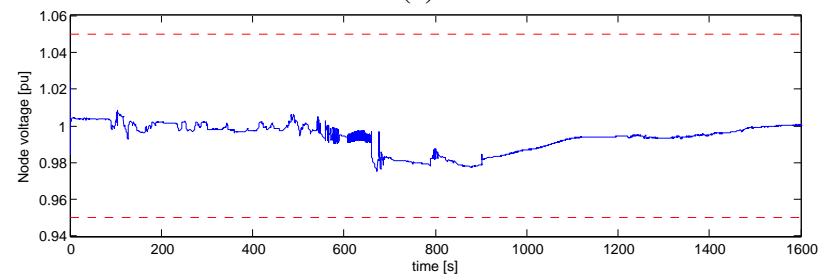

(d)

Fig. 3: COMMELEC control strategy: WB2 power consumption (a), PV3 production (b), line current (c) and node voltage (d).

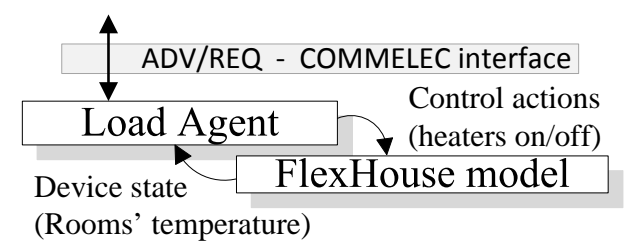

Fig. 4: Interaction between the Load Agent and the building simulator.

the control algorithm used in the LA to implement the power setpoint requested by the GA.

\section{A. PQt profile}

The Load Agent advertises to the GA the region in the $(P, Q)$ plane of the load admissible active and reactive power setpoints at time $t$, where negative power means consumption. Being $\mathcal{X}$ the set of all possible power setpoints $x=$ $(P, Q) \in \mathbb{R}^{2}$, the $P Q t$ profile sent at time $t_{0}$ is the collection: $\mathcal{A}(t) \subseteq \mathcal{X}$, where $t$ is the time instant at which the $P Q t$ profile is advertised. Since in this study the controlled system consists of resistive space heaters, only pure active power consumption is considered in a way that $\mathcal{A}$ is in this case an interval in $P$ and zero in $Q$. 
Even if the notion of the load flexibility is associated to both the PQt profile and the cost function, we here link it to the former. Namely, the load is flexible if it can be interrupted and re-started without causing user discomfort or device failure. Specifically, a single room flexibility is determined by the fact that the heater can be switched on or off without compromising the user comfort as long as the room temperature is within the comfort bounds. Conversely, if the room temperature is below the comfort bound $\left(T_{L B}\right)$ the heater must operate and if the temperature is above $\left(T_{U B}\right)$ the heater must not operate (Fig. 5).

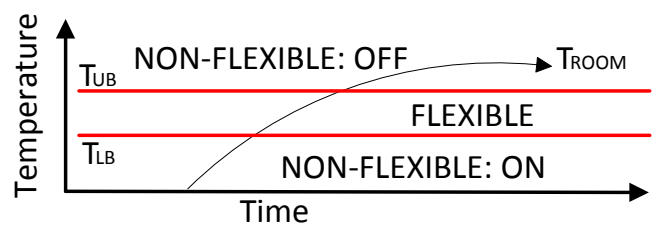

Fig. 5: The flexibility of operation of a heating system is related to the system state itself.

The flexibility of the whole system (building) is determined by its subsystems (rooms) flexibilities. Let $\mathcal{R}$ denote the set of rooms in the building and $j \in \mathcal{R}$ the room index. Define $\mathcal{F}_{j}$ as the flexibility of room $j$ in terms of upper and lower active power consumption:

$$
\mathcal{F}_{j}=\left\{\begin{array}{ll}
{\left[P_{j}^{*}, P_{j}^{*}\right] \quad: \quad T_{j}<T_{L B, j}} \\
{\left[P_{j}^{*}, 0\right]: \quad T_{L B, j} \leq T_{j} \leq T_{U B, j} \quad,} \\
{[0,0]: \quad T_{j}>T_{U B, j}}
\end{array},\right.
$$

where $P_{j}^{*}$ is the rated active powers of the heating system of room $j$.

The building $P Q t$ profile is obtained by performing the Minkowski sum of $\mathcal{F}_{j}$ over $\mathcal{R}$ :

$$
\mathcal{A}=\sum_{j \in \mathcal{R}} \mathcal{F}_{j}
$$

The rooms whose temperature is below $T_{L B}$ determine the building minimum power consumption, while the maximum consumption is limited by those rooms whose temperature is above $T_{U B}$. The $(P, Q)$ flexible region is determined by those rooms which temperature is within the comfort requirements $\left[T_{L B}, T_{U B}\right]$.

Figure 6 presents the computation of the flexibility region based on rooms temperatures in case of a linear variation of external temperature. It is possible to observe the effect of this disturbance on the $P Q t$ profile.

The example provided in Fig. 6 aims at showing how the upper and lower bounds of set $\mathcal{A}$ evolve with respect to the system state and how external disturbances impact the system flexibility. It can be seen that the bounds of $\mathcal{A}$ coincide as soon as the rooms are outside the comfort zone (from time 0 to 0.2 hours) and load consumption constrained to $8 \mathrm{~kW}$. Analogous situation occurs when, due to an external disturbance in the outside temperature, the rooms' temperature rises above $T_{U B}$, causing the load consumption to be constrained to zero.
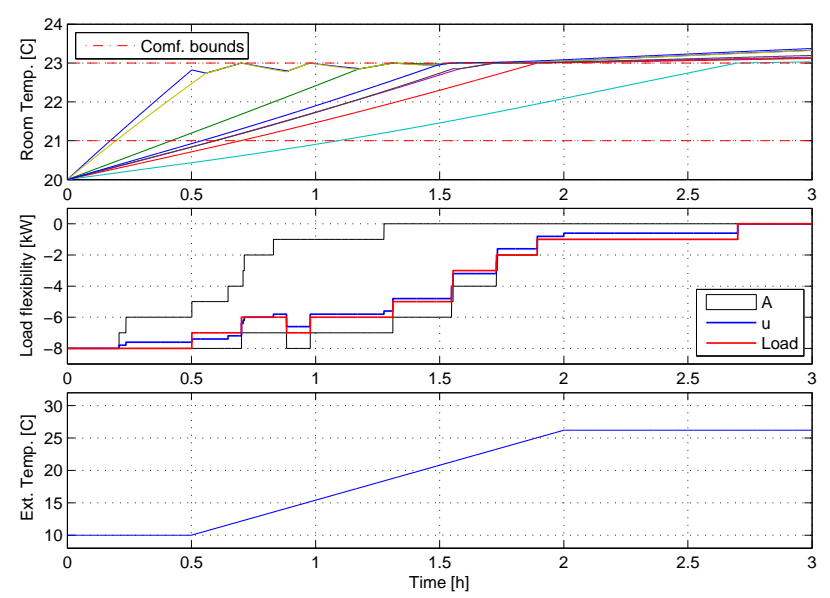

Fig. 6: Evolution of rooms' temperature (first sub-plot), $P Q t$ profile computation (second sub-plot) and external temperature (third sub-plot).

In the same figure, the blue line is the power setpoint from the GA and the red line is the load consumption. The LA implements the requested power setpoint by means of Laod Admission Control (LAC), which details are deferred to Sec. III-D.

\section{B. Belief function}

The belief function, henceforth denoted by $B$, describes the ability of the Load Agent to map a requested power setpoint, $u$, coming from the Grid Agent, to actual load consumption. Note that $B$ is a set-valued function from $\mathbb{R}^{2}$ to sets over $\mathbb{R}^{2}$ and is designed to account for model uncertainties and quantization effects in the load.

In this study, the building is composed by eight rooms with one electric space heater each. The heaters can be either switched on or off, therefore the load can implement only discrete active power setpoints from $0 k W$ to $8 k W$ in steps of $1 \mathrm{~kW}$. Hence the belief function simplifies to the nearest integer function:

$$
B(u)=\{\operatorname{nint}(u)\}
$$

where $u$ is a power setpoint received from the GA.

Note that, thanks to the LAC algorithm (Sec. III-D), the LA achieves tracking a power setpoint from the GA with an accuracy of $\pm 0.5 \mathrm{~kW}$.

\section{Virtual cost function}

The cost function serves as a proxy for load internal conditions and it associates a cost to every operating point $x \in \mathcal{A}$ and it is a map: $C: \mathcal{A} \mapsto \mathbb{R}$, that is formally defined as:

$$
C(x)=\sum_{j \in \mathcal{R}}\left[U(j) c_{h}\left(T_{j}\right)-(1-U(j)) c_{h}\left(T_{j}\right)\right],
$$

where the terms $U(j)$ are the components of the heaters activation vector $\vec{U}$. This vector, given a certain state of the system, is computed with respect to a specific operating point $x$ by means of LAC (Sec III-D): $\vec{U}=L A C(\mathcal{M}, x)$. 
In Eq. (4) the terms $c_{h}\left(T_{j}\right)$ are computed as:

$$
c_{h}\left(T_{j}\right):\left\{\begin{array}{cl}
1+\left(T_{j}-T_{U B_{j}}\right)^{2} & : T_{j} \geq T_{U B_{j}} \\
\frac{T_{j}-T_{M_{j}}}{T_{U B_{j}}-T_{M_{j}}} & : T_{L B_{j}}<T_{j}<T_{U B_{j}} \\
-1-\left(T_{L B}-T_{j}\right)^{2} & : T_{j} \leq T_{L B_{j}}
\end{array}\right.
$$

and they express the cost associated to heating a room $j ; T_{j}$ is the room internal temperature, $T_{U B_{j}}$ and $T_{L B_{j}}$ are the comfort upper and lower bounds and $T_{M_{j}}=\left(T_{U B_{j}}-T_{L B_{j}}\right) / 2$.

Equation (5) returns zero when the room temperature is in the middle of the comfort band, $T_{M}$, positive when it is above $T_{M}$ and negative when it is below. This design choice follows the fact that the building exhibits the maximum flexibility for longer time if all the rooms temperatures are in the middle of their comfort zone. The cost is linearly defined within the comfort zone in order to not excessively penalize deviations from $T_{M}$ and it is always related to the action of heating $(U(j)=1)$. It follows that the action of not heating $(U(j)=$ $0)$ generates opposite cost in Eq. (4). Therefore the total cost expressed in Eq. (4) is obtained by summing up the costs related to each room control action. Figure 7 shows examples of virtual costs in correspondence of different times

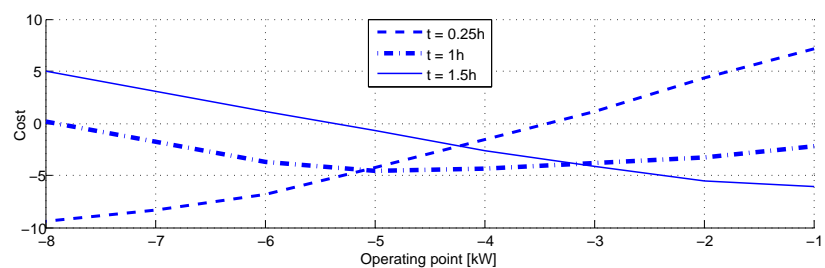

Fig. 7: Example of cost function computed for different system states $(t=$ $0.25 h, t=1 h, t=1.5 h)$

Note that in this case study only active power operating points are considered and the LA does not need any information about the building model to compute the cost. Every time the Load Agent computes the cost function, it uses the information on load state (temperatures) to compute the terms $c_{h}$ and it calls the $L A C$ algorithm, presented in the next subsection, which uses the same temperature and comfort information to compute the $U(j)$.

\section{Load Admission Control (LAC) algorithm}

This subsection presents the algorithm for load Direct Power Control (DPC) via Load Admission Control (LAC). DPC is introduced in [5] and consists of run-time control of a DER for the tracking of a power setpoint. It differs from the Power Schedule by the fact that future values of the power setpoint are not known to the DER. The interested reader can refer to [6] and the references therein for an overview on control policies for energy consumption in buildings.

Algorithm 1 presents the Load Admission Control, an heuristic-based search algorithm for load online scheduling that was first introduced in [7] for demand response applications and provision of PowerMax service [5] . However, the algorithm presented in [7] is here modified in order to track a power reference instead of limiting the peak power.
Define the triple $\gamma_{j}$ associated to room $j$ as:

$$
\gamma_{j}=\left\langle h_{j}, P_{j}^{*}, p_{j}\right\rangle
$$

where $P_{j}^{*}$ is the heater rated active power consumption, $h_{j}$ is a priority factor defined as:

$$
h_{j}= \begin{cases}1 & : T_{j} \leq T_{L B_{j}} \\ \frac{T_{j}-T_{L B, j}}{T_{U B_{j}}-T_{L B, j}} & : T_{L B_{j}}<T_{j}<T_{U B_{j}} \\ 0 & : T_{j} \geq T_{U B_{j}}\end{cases}
$$

and $p_{j}$ is boolean value representing the load preemptiveness (interruptibility). A device is interruptible if its operation state can be modified from $\mathrm{ON}$ to OFF. Heaters in rooms which temperature is below $T_{L B}$ are non-preemptible.

Define $(\mathcal{M}, \leq)$ a totally ordered set of pending consumption requests:

$$
\mathcal{M}=\bigcup_{j \in \mathcal{R}} \gamma_{j},
$$

where $\gamma_{k} \leq \gamma_{j}, \forall k>j$ and $(\leq)$ is a Lexicographical order.

Given a power setpoint from the GA, $u$, the control vector $\vec{U}$ for the heaters is computed via LAC: $\vec{U}=L A C(\mathcal{M}, u)$, where $L A C(\cdot)$ is detailed in Algorithm 1.

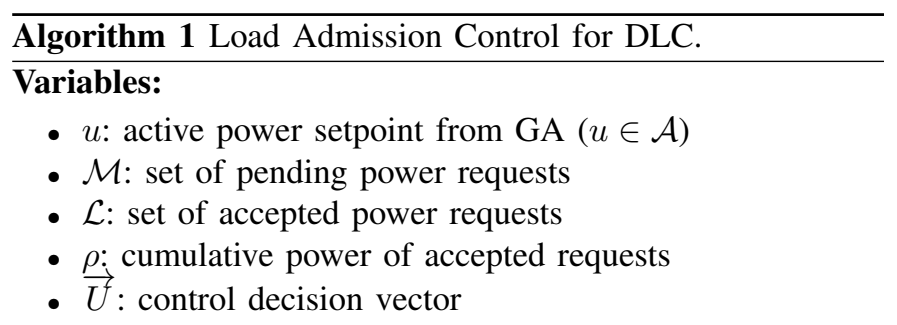

\section{Require:}

Initialize: $\mathcal{L}=\emptyset, \rho=0, U=O$

for all $\gamma_{j} \in \mathcal{M}$ do

if $p_{j}=$ false then

$\mathcal{M}=\mathcal{M} \backslash \gamma_{j}, \mathcal{L}=\mathcal{L} \cup \gamma_{j}, \rho=\rho+P_{j}^{*}$

$$
\text { end if }
$$

\section{end for}

$$
\begin{aligned}
& \text { for all } \gamma_{j} \in \mathcal{M} \text { do } \\
& \quad \text { if }\left|u-\rho-P_{j}^{*}\right|<= \\
& \quad \mathcal{M}=\mathcal{M} \backslash \gamma_{j}, \mathcal{L} \\
& \text { end if } \\
& \text { end for } \\
& \text { set } U(j)=1 \forall \gamma_{j} \in \mathcal{L} \\
& \text { return } \vec{U}
\end{aligned}
$$$$
\text { if }\left|u-\rho-P_{j}^{*}\right|<=|u-\rho| \text { then }
$$$$
\mathcal{M}=\mathcal{M} \backslash \gamma_{j}, \mathcal{L}=\mathcal{L} \cup \gamma_{j}, \rho=\rho+P_{j}^{*}
$$

\section{Simulation}

This section presents two simulations studies that show the building response to signals from the LVGA when: i) six rooms out of eight are initialized below the comfort zone (Sec. IV-A), ii) all the rooms are initialized within the comfort zone (Sec. IV-B). Note that the LVGA here was not modified with respect to the simulation presented in Sec. II. 


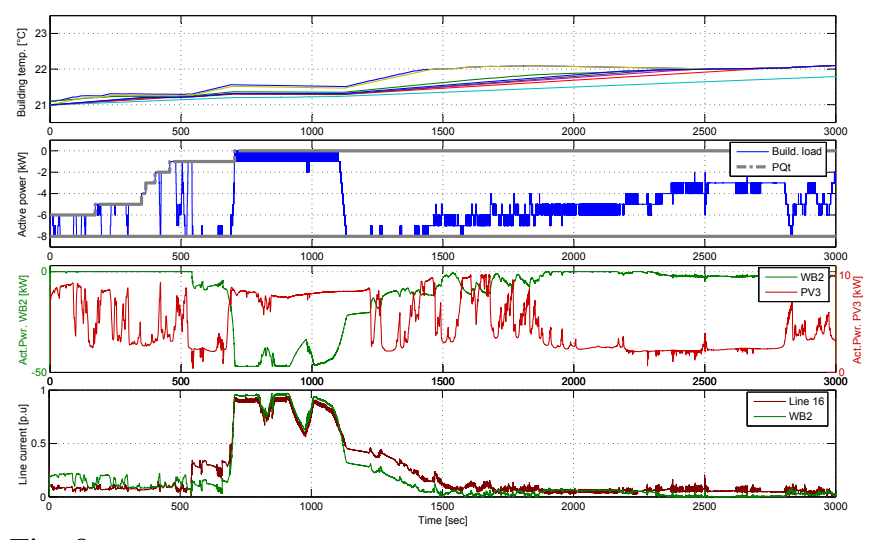

Fig. 8: Simulation with LA - rooms initialized below the comfort zone. From the top chart: temperature of building rooms, building power consumption WB2 consumption and PV3 production, building and connecting line (Line 16) currents.

\section{A. First scenario: load initialized below the comfort zone}

In this simulation, the $P Q t$ profile of the LA is initially between $-6 k W$ and $-8 k W$, as six rooms out of eight are below the comfort zone (Fig. 8). The LA is able to track the explicit power setpoint coming from the LVGA and the building flexibility increases as soon as all the rooms enter the comfort zone (upper chart). At this time, the LVGA fully exploits the load flexibility when dealing with local resource contingencies and line constraints.

One can observe that, given a combination of such events as: increased PV production, increased building flexibility and pending WB2 operation, the LVGA allows the WB2 to charge (by increasing the WB2 power setpoint) and limits the building consumption (by decreasing the LA power setpoint) in order to avoid the congestion of the line connecting the water boiler, the building and the two PV installations to the distribution feeder (Line 16 in Fig. 1). As intrinsic property of composability of the system, this behaviour emerges without the need of any predefined control policy or manual intervention.

The building is used as a virtual electrical storage resource by the LVGA to optimize the operation of the microgrid, as the LA is requested to partially follow the local PV production (PV2 receives the same irradiation of PV3). At the same time, as the rooms approach the midpoint of the comfort zone, due to the virtual cost function, the LVGA reduces the heating input (Fig. 8, last part of the simulation).

\section{B. Second scenario: load initialized within the comfort zone}

Due to the initialization of the internal temperature in all the rooms within the comfort zone, the $P Q t$ profile is constrained between $0 k W$ and $-8 k W$ (Fig. 9), in a way that the building exhibits the maximal flexibility to the LVGA from the beginning.

Figure 9 shows that only the rooms in the lower part of the comfort zone are heated, while the LVGA operates the water boiler WB2 in advance with respect to the previous simulation. When WB2 is switched on, the LA is requested to reduce the building consumption in order to not overload the feeder. At about 1200s, the decreased PV production brings the LVGA

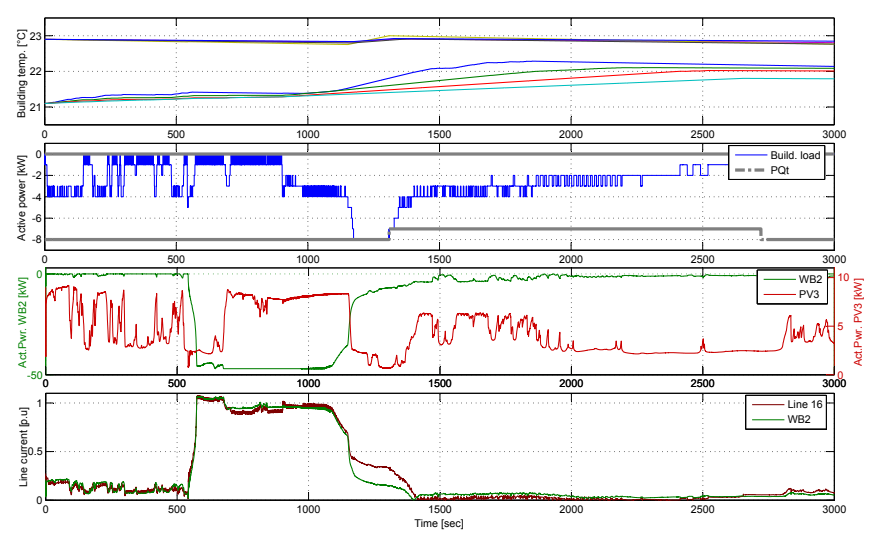

Fig. 9: Simulation with LA - rooms initialized within the comfort zone. From the top chart: temperature of building rooms, building power consumption, WB2 consumption and PV3 production, building and connecting line (Line 16).

to reduce the WB2 charging power and restore the building heating so that all the rooms steer toward the middle of the comfort zone (i.e. to the minimum virtual cost).

\section{CONCLUSIONS AND FUTURE WORK}

This paper presented a systematic approach to abstract the flexibility of a building heating system and interface it with the composable framework for real-time grid control, COMMELEC, i.e. to be able to use a building's thermal inertia for real-time control.

The presented simulations show the efficacy of the LA to operate the load within the user comfort requirements and communicate generic information to the GA to perform explicit power control. The LA designed for the building space heating system is independent from the building model and it was plugged in the COMMELEC framework and tested in the same setup presented in [2]. In this context, the next research steps foresee the implementation of the presented LA in a realtime control platform and test with real loads in a microgrid setup currently developed at EPFL.

\section{REFERENCES}

[1] K. A. Papadogiannis and N. Hatziargyriou, "Optimal allocation of primary reserve services in energy markets," Power Systems, IEEE Transactions on, vol. 19, no. 1, pp. 652-659, Feb 2004.

[2] A. Bernstein, L. Reyes-Chamorro, J.-Y. Le Boudec, and M. Paolone, "A composable method for real-time control of active distribution networks with explicit power setpoints," 2014, arXiv:1403.2407.

[3] S. Papathanassiou, N. Hatziargyriou, K. Strunz et al., "A benchmark low voltage microgrid network," in Proceedings of the CIGRE Symposium: Power Systems with Dispersed Generation, 2005, pp. 1-8.

[4] D. E. M. Bondy and J. Parvizi, "Modeling, identification and control for heat dynamics of buildings using robust economic model predictive control," Master's thesis, Technical University of Denmark, 2012.

[5] O. Gehrke and F. R. Isleifsson, "An aggregation friendly information model for demand side resources," in 2010 IEEE 35th Conference on Local Computer Networks, pp. 1019-1023.

[6] A. Kosek, G. Costanzo, H. Bindner, and O. Gehrke, "An overview of demand side management control schemes for buildings in smart grids," in Smart Energy Grid Engineering (SEGE), 2013 IEEE International Conference on, Aug 2013, pp. 1-9.

[7] G. Costanzo, J. Kheir, and G. Zhu, "Peak-load shaving in smart homes via online scheduling," in Industrial Electronics (ISIE), 2011 IEEE International Symposium on, June 2011, pp. 1347-1352. 